\title{
Selective Manipulation of Microscopic Particles with Precursor Swirling Rayleigh Waves
}

\author{
Antoine Riaud, ${ }^{1,2}$ Michael Baudoin, ${ }^{1, *}$ Olivier Bou Matar, ${ }^{1}$ Loic Becerra, ${ }^{2}$ and Jean-Louis Thomas ${ }^{2, \dagger}$ \\ ${ }^{1}$ Univ. Lille, CNRS, Centrale Lille, ISEN, Univ. Valenciennes, UMR 8520, \\ International Laboratory LEMAC/LICS - IEMN, F-59000 Lille, France \\ ${ }^{2}$ Sorbonne Universités, UPMC Univ Paris 06, CNRS UMR 7588, Institut des NanoSciences de Paris, \\ 4 place Jussieu, 75006 Paris, France \\ (Received 18 July 2016; revised manuscript received 15 November 2016; published 8 February 2017)
}

\begin{abstract}
Contactless manipulation of microparticles is demonstrated with single-beam acoustical tweezers based on precursor swirling Rayleigh waves. These surface waves degenerate into acoustical vortices when crossing a stack made of a fluid layer and its solid support, hence creating a localized acoustical trap in a fluid cavity. They can be synthesized with a single interdigitated transducer whose spiraling shape encodes the phase of the field like a hologram. For applications, these tweezers have many attractive features: they are selective, flat, easily integrable, and compatible with disposable substrates.
\end{abstract}

DOI: 10.1103/PhysRevApplied.7.024007

\section{INTRODUCTION}

High-precision contactless manipulation offers tremendous perspectives for mesoscale physics and biophysical studies [1]. A large span of methods using magnetic [2,3], optical [4,5], electrical [6], and acoustical forces [7-10] and their combinations [11] have been investigated in recent decades. Among these techniques, acoustic tweezers stand out for cell-manipulation applications, as they combine large forces [12,13], high biocompatibility [14-16], label-free manipulation [17], and disposable parts for minimized sample contamination [18]. Compared to their optical counterpart, a major advantage of acoustical tweezers is that several fewer orders of magnitude power is required to apply the same force on particles. Indeed, the radiation pressure used for trapping is proportional to $I / c$ in both acoustics and optics, where $I$ is the wave intensity and $c$ the wave speed $\left(\simeq 1500 \mathrm{~ms}^{-1}\right.$ for an acoustic wave in water and $\simeq 3 \times 10^{8} \mathrm{~ms}^{-1}$ for light). This efficiency limits spurious heating, which can be detrimental for cell or microorganism manipulation. Moreover, acoustical tweezers also offer an alternative to optical tweezers for experiments in opaque media or when manipulating light-sensitive samples.

Technologies based on standing surface acoustic waves (SAWs) allow collective contactless manipulation of microobjects. For instance, Ding et al. [19] and Tran et al. [20] independently proposed similar devices where two orthogonal standing SAWs create an egg-box potential well network where numerous particles can be captured and translated at once. Standing SAW traps are flat devices that are easily miniaturized [21], and they benefit from the standard fabrication process by photolithography and metal sputtering. Although these devices are highly suitable for massively parallel experiments, the simple nature of the

\footnotetext{
*michael.baudoin@univ-lille1.fr †jean-louis.thomas@upmc.fr
}

acoustic field generated by this kind of device is unsuitable for selectively manipulating one particle within a cluster.

One approach for capturing individual particles relies on highly focused Gaussian ultrasonic beams [22]. This system gives promising results for biophysical applications [23-25] but has been restricted thus far to trapping cells and particles on Mylar films, which is not a standard culture medium. Furthermore, it was shown that, in open media, cells tend to migrate towards pressure nodes [26-28] and, therefore, require an acoustic beam with a dark focus [29]. In this regard, dexterous and selective contactless manipulation was recently achieved [13,30,31] using acoustical vortices. These helical waves spin around a phase singularity where wave-front dislocation yields a total cancellation of the beam amplitude [32]. This dark spot is wrapped by a bright ring of maximum sound intensity which, in turn, enables a selective trapping at this dark focus [29]. The synthesis of acoustical vortices is challenging [32]: it requires most often a network of numerous transducers controlled by a high-end programmable electronics [33]. Such a configuration is advantageous for synthesizing various types of fields [30] or digitally controlling the position of the acoustical trap [31]. Nonetheless, the large number of individual transducers makes the miniaturization challenging, and the electronic hardware causes the device cost to skyrocket. Furthermore, these devices and their successive embodiments [34-36] are not flat, which complicates integration with other microfluidic elements.

One possibility for overcoming these limitations is to use surface acoustic waves. Recently, we expanded the surfaceacoustic-wave toolbox [37] with the 2D analog of acoustical vortices called swirling SAWs [38]; these waves, in turn, generate acoustical vortices when immersed in a liquid [39]. Similar to their 3D analog, the experimental synthesis of swirling SAWs is challenging and thus far involves an array of 32 interdigitated transducers (IDTs) controlled independently by a high-end programmable 
electronics [38] and the inverse-filtering technique to determine the signal emitted by each transducer.

In this paper, we introduce an alternative class of surface waves called "precursor swirling Rayleigh" (PSR) waves that are designed to degenerate into acoustical vortices after crossing a stack made of a liquid layer and its solid support, hence creating a localized particle trap in a fluid cavity. PSR waves are defined through the resolution of a complex inverse problem involving wave synthesis with the piezoelectric effect and Bessel-beam degeneration. We demonstrate that a single spiraling IDT encodes the wave like a hologram, which enables faithful generation of the targeted acoustical vortex. This flat technology therefore does not require any external acoustical-wave source and can be easily miniaturized and integrated in a MEMS or in a lab-ona-chip. We also predict numerically and confirm experimentally the force applied by the acoustic radiation pressure at the core of these vortices on $30-\mu \mathrm{m}$ polystyrene (PS) particles. Finally, the potential of these tweezers for selective particle manipulation is demonstrated by patterning individually several dozen particles that are initially dispersed randomly in a microfluidic chamber. These spiraling IDTs constitute the core of an alternative type of acoustic tweezers able to generate acoustical vortices at remote locations through one or several layers of materials.

\section{THEORETICAL ANALYSIS}

Surface acoustic waves are mechanical vibrations propagating at the surface of a generally anisotropic substrate with a slowness $s_{\mathrm{SAW}}(\psi)$ that depends on the direction of propagation $\psi$. This dispersion relation restricts the variety of fields that can be synthesized. Therefore, any surface acoustic wave can be written as follows in the complex space [38]:

$$
\Xi^{(0)}=e^{i \omega(t-\tau)} \int_{-\pi}^{\pi} \mathcal{H}^{(0)}(\psi) e^{-i \omega h(\psi, \theta) r} d \psi,
$$

where $\Xi^{(0)}$ is the normal vibration amplitude of the substrate at the point $(r, \theta)$ in polar coordinates and at time $t, \omega$ the wave frequency, and $h$ a function defined by $h(\psi, \theta)=s_{\mathrm{SAW}}(\psi) \cos (\psi-\theta)$. Finally, $\mathcal{H}^{(0)}$ is the angular spectrum of the wave, that is, the relative weight of the plane waves for each direction $\psi$. It is the sole degree of freedom in Eq. (1). For the subsequent discussion, we introduce the complex phase $\mu$ and the amplitude $\xi$ of $\mathcal{H}^{(0)}(\psi)=\xi(\psi) e^{i \mu(\psi)}$ and a time delay $\tau$. In this paper, we consider only surface waves propagating on substrates whose thickness is much larger than the wavelength. These surface waves are called Rayleigh waves.

At the surface of piezoelectric materials, surface acoustic waves combine periodical displacements in the three directions of space and electrical oscillations, the proportion of each displacement being $\psi$ dependent. In the following, we refer to $a(\psi)$ as the normal substrate vibration amplitude per unit voltage ( $a$ and $s_{\mathrm{SAW}}$ can be computed by a range of methods [40,41] and are provided in the Supplemental Material [42]). Conversely, this fundamental electromechanical coupling ensures that forcing an alternating current through a piezoelectric substrate induces periodical deformations of the solid. This action can be achieved with socalled IDTs, two interlocking arrays of metallic electrodes of inverse polarity which reinforce the vibrations by constructive interference. The distance between these metallic fingers is chosen to match with the wavelength of the targeted SAW. For a plane wave, this condition is simple since the wavelength is constant in a given wave-propagation direction. It is, nonetheless, more challenging to fulfill this resonance condition for more-complex wave fields. Since no mathematical background is currently available to design a suitable IDT for the synthesis of any surface acoustic wave compatible with the substrate anisotropy, the use of these systems has been limited thus far to the synthesis of plane waves and, in very few cases, focalized waves [43]. In the Appendix, we derive a general formula of the IDT's shape for the synthesis of any arbitrary SAW field $\Xi^{(0)}$. In our calculations, the positive and negative electrodes run along a polar curve $R(\Omega)$ and are exposed to an alternating voltage $V(\Omega)$ :

$$
\begin{array}{r}
V(\Omega)=e^{i \omega t} \frac{\xi(\bar{\psi}+\pi) \sqrt{2 \pi}}{|a(\bar{\psi})| \sqrt{R(\Omega) \omega\left|h^{\prime \prime}(\bar{\psi}, \Omega)\right|}}, \\
R(\Omega)=\frac{\psi_{0}+\alpha(\bar{\psi})-\frac{\pi}{4} \operatorname{sgn}\left[h^{\prime \prime}(\bar{\psi}, \Omega)\right]-\mu(\bar{\psi}+\pi)}{\omega h(\bar{\psi}, \Omega)},
\end{array}
$$

where $\psi_{0}=\omega \tau+b \pi$ for the positive electrode and $\psi_{0}=$ $\omega \tau+(b+1) \pi$ for the negative one and $b \in \mathbb{Z}$, a parameter introduced for symmetry reasons [see Eqs. (A6) and (A7) in the Appendix]. $\alpha(\bar{\psi})$ is the complex argument of $a(\bar{\psi})$. In this equation, the beam stirring angle $\Omega-\bar{\psi}(\Omega)$ is defined by $h^{\prime}(\bar{\psi})=0$ with $h^{\prime}=\frac{\partial h}{\partial \psi}$. As pointed out by Laude et al. [43], $h(\bar{\psi}, \Omega)$ is related to the group velocity $v_{g}(\Omega)=1 / h(\bar{\psi}, \Omega)$. The term $\sqrt{\left|h^{\prime \prime}(\bar{\psi}, \Omega)\right|}$, proportional to the phonon focusing factor, may vanish for some material cuts in certain directions, in which case it yields cuspidal points and caustics. In Eqs. (2) and (3), the $\pi$ offset of the $\mu$ and $\xi$ arguments represents the fact that the electrode located at $\Omega$ generates a plane wave propagating with a direction $\bar{\psi}(\Omega)+\pi$. The term $\sqrt{R}$ accounts for the antenna gain of the transducer. Although $R$ is fairly easy to calculate and tune, $V$ (the excitation magnitude of a specific IDT portion) mostly depends on $a(\psi)$, which is specific to the material and its cut (see also Ref. [44]). Setting $V$ may involve the ability to apply different voltage magnitudes on different finger pairs or increasing the number of finger pairs in the directions of weaker coupling $a(\bar{\psi})$.

\section{EXPERIMENTAL SYNTHESIS}

An exciting application of this inverse problem is the synthesis of acoustical vortices with swirling SAWs (their 
2D analogs), which, in turn, may achieve selective contactless manipulation of microparticles and biological cells (see Fig. 2). According to earlier studies [38], it is not possible to generate isotropic swirling SAWs in anisotropic piezoelectric substrates but rather their anisotropic counterpart only, as defined by

$$
\mathcal{W}_{l}=\frac{i^{l} e^{i \omega t}}{2 \pi} \int_{-\pi}^{\pi} \rho(\psi) e^{-i l \psi-i \omega h(\psi, \theta) r} d \psi,
$$

where $l$ is the topological charge of the vortex and $\rho(\psi)$ is the amplitude of the wave in a given direction. These anisotropic swirling SAWs degenerate when they are transmitted and then propagate in an isotropic medium [39]. This degeneration can be controlled by synthesizing adequate precursor waves of angular spectrum $\mathcal{H}^{(0)}$ on the substrate that degenerate into the desired field of angular spectrum $\mathcal{H}^{(n)}$ after crossing $n$ possibly anisotropic superstrates. According to the angular-spectrum propagation calculations in the Appendix, we get

$$
\begin{gathered}
\mathcal{H}^{(0)}=\mathcal{H}^{(n)} e^{i \omega \mathcal{T}}, \\
\text { with } \mathcal{T}=\sum_{i=1}^{n} s_{\mathcal{Z}}^{(i)}\left(z_{i}-z_{i-1}\right), \\
s_{Z}^{(i)}=\sqrt{s^{(i) 2}-s_{\mathrm{SAW}}{ }^{2}},
\end{gathered}
$$

and, in the case of acoustical vortices,

$$
\mathcal{H}^{(n)}=\frac{i^{l} \rho(\psi) e^{-i l \psi}}{2 \pi} .
$$

$e^{i \omega \mathcal{T}}$ is the propagator, where $\mathcal{T}$ represents the directiondependent delay attributable to the propagation across $n$ superstrates of slowness $s^{(i)}$, with $i \in\{1, \ldots, n\}$ and their interface located at $z_{i}$. Equation (7) is restricted to isotropic superstrates (the general case is detailed in the Appendix). In order to create the acoustical vortex given by Eq. (4), we generate the precursor wave whose angular spectrum is given by Eqs. (5)-(8). This wave is, in turn, synthesized by the transducer described by Eq. (3). These calculations are implemented in the PYTHON code provided in the Supplemental Material [42]. Importantly, Eqs. (3) and (5) show that $R$ depends on the frequency of actuation, the slowness of the piezoelectric substrate and the superstrates, and the thickness of each superstrate layer. Accordingly, each transducer has to be designed specifically for this set of parameters. This requirement is not very restrictive since standardized microscope slides and coverslips made of glass and other materials are readily available. Remarkably, the final geometry of the transducer seems suitable for superharmonic generation. Accordingly, harmonic $m$ is expected to generate an acoustical vortex of charge $l m$ when excited at a pulsation $\omega_{m}=m \omega$ (see the proof in the Supplemental Material [42]).
In the first set of experiments, we fabricate the transducer shown in Fig. 1(a) to form clockwise acoustical vortices $\mathcal{W}_{1}$ across a typical glass coverslip used in microscopy applications (no. 1 borosilicate, thickness $150 \mu \mathrm{m}$ ). The lithography process and the masks are available in the Supplemental Material [42]. The transducer twists positive and negative electrodes in a double Archimedean spiral. The diameter of the device is constrained by the attenuation of a leaky surface wave that is approximately $2 \mathrm{~dB} / \mathrm{MHz}$ $\mathrm{cm}$. The positive and negative electrodes are connected to opposite supply branches, with the branches themselves being located in the minimal piezoelectric coupling directions. The working frequency of this specific device is $10 \mathrm{MHz}$, which corresponds to wavelengths near $400 \mu \mathrm{m}$ and optimal particle size range close to $70 \mu \mathrm{m}[13,21]$. Standard lithography workbench allows resolutions down to $1 \mu \mathrm{m}$, which lets us envision the manipulation of submicrometric objects with a similar setup.

In order to measure the vertical vibrations created by this transducer after crossing the glass coverslip, we coat it with a thin layer of gold. We then measure the vertical vibration amplitude of the substrate [see Figs. 1(b) and 1(c)] using a homemade vibrometer described in Ref. [38]. The experiment is as follows. We drop a small volume of silicon oil (1.0 Pas) directly on the transducer surface and then squeeze it with a gold-coated coverslip. The silicon oil is used as a coupling fluid for the transmission of the acoustic wave from the piezoelectric transducer to the

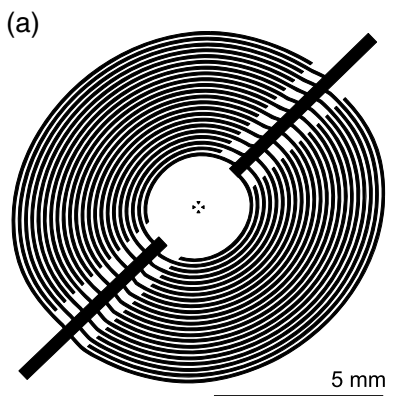

(c)

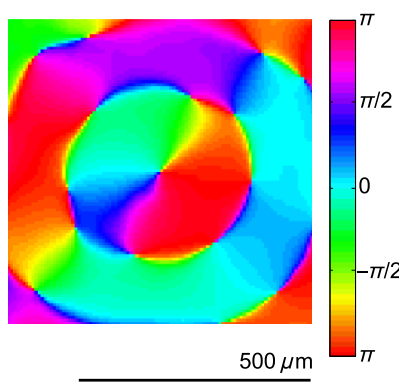

(b)

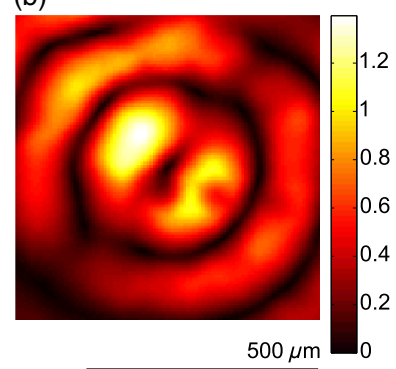

(d)

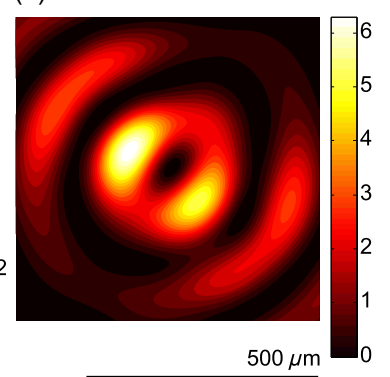

FIG. 1. (a) Spiraling IDT for precursor swirling Rayleigh (PSR) wave synthesis (clockwise $\mathcal{W}_{1}$ at $10 \mathrm{MHz}$ across no. 1 glass coverslip). (b) Experimental substrate vertical vibration amplitude (max amplitude $1.4 \mathrm{~nm}_{\mathrm{pp}}$ at $7 \mathrm{~V}_{\mathrm{rms}}$ ). (c) Experimental substrate vertical vibration phase. (d) Calculated potential well from experimental data (max height $6.3 \mathrm{fJ}$ ). 
substrate. Finally, we add a small droplet of water and cap it with a 2-mm-thick block of cross-linked polydimethylsiloxane (PDMS), a highly efficient acoustic absorber for minimizing acoustic reflections. The Michelson vibrometer then measures the vibrations of the top surface of the coverslip. The wave measured at this position is representative of the wave inside the water layer since it is only a few wavelengths high. This assertion can be checked in the simulation shown in Fig. 7 of the Supplemental Material [42]. Accordingly, the vortex diameter sizes roughly $\lambda / 2=175 \mu \mathrm{m}$. The phase singularity shown in Fig. 1(c) is clearly visible and validates the calculation of $R$. It results directly in the dark core in the center of Fig. 1(b). Knowing the vertical vibration amplitude (about $1.4 \mathrm{~nm}_{\mathrm{pp}}$ at $7 \mathrm{~V}_{\mathrm{rms}}$ ), it is possible to compute the force applied by this vortex on spheres contained in the thin water layer between the substrate and the PDMS. In the case of small particles, this force is the gradient of the potential $[27,28,45]$ shown in Fig. 1(d). The theoretical calculation and maximum displacement speed experiments (details in the Supplemental Material [42]) agree quantitatively well on a maximum force of $200 \mathrm{pN}$ on $30-\mu \mathrm{m}$ PS spheres at $7 \mathrm{~V}_{\mathrm{rms}}(1 \mathrm{~W})$, which is the upper bound of optical tweezers [5]. As in previous studies on acoustical vortex traps [13], this force is attained with a maximum power density of $0.15 \mathrm{~W} / \mathrm{cm}^{2}$, more than 4 orders of magnitude below the $2.3 \mathrm{~kW} / \mathrm{cm}^{2}$ [46] required by optical tweezers for a similar trap.

These acoustical tweezers are integrated into a microscope for particle manipulation. The manipulation setup [see Fig. 2(a)] combines a static part (the transducer) and a mobile part (the substrate) displaced with a manual stage. For practical reasons (simpler electrical connections and visualization), the transducer is fixed in the laboratory frame and the sample is made mobile by the means of a micromanipulator. Particle position recording is achieved with a Flea 3 camera (PointGrey) mounted on an MZ1 microscope (ViewSolution). Polystyrene spheres of $30 \mu \mathrm{m}$ diameter (Themoscientific) are displaced sequentially by placing the transducer below one of them, turning the power on, moving the transducer and the captured object to the desired location, then turning the power off again to release the particle (see Video 1 and the related video in the Supplemental Material [42]). In this process, the bright ring creates a circular potential barrier that maintains the target particle at the center of the vortex but also repels its neighbors outwards. As a consequence, two adjacent particles can be manipulated independently only if their distance exceeds half an acoustic wavelength, $\lambda_{\text {SAW }}$. Furthermore, the vortex comprises several secondary rings of decreasing intensity that also exert forces on particles. The selectivity of this tweezer thus relies (i) on the larger trapping force applied on the particles at the center of the vortex compared to the secondary rings and (ii) on the combination of the tweezer with substrates of suitable friction coefficients that creates a threshold for the particle

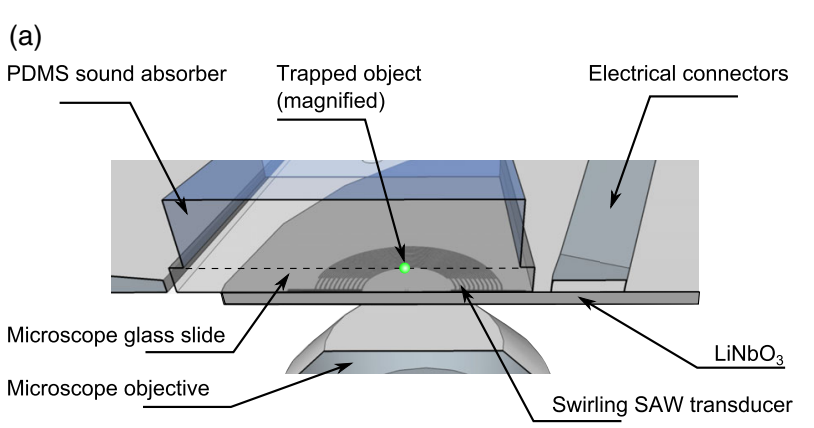

(b)



FIG. 2. PSR-based acoustical tweezers integrated in a microscope for particle manipulation. The transducer is a pair of spiraling interdigitated gold electrodes deposited on a piezoelectric substrate. A microscope slide is placed on the transducer, and acoustic transmission is ensured by the mean of a thin layer of silicon oil. An aqueous suspension of micrometric polystyrene beads is dispersed between the microscope slide and an upper layer of PDMS that plays the role of acoustic absorber. The center of the vortex is visualized by a reticule. The device is represented (a) at the top, while (b) the bottom provides a more schematic cross section.

displacement. Thus, we switch from borosilicate glass to 2-mm-thick polymethyl methacrylate (PMMA) slides, which exhibit larger friction coefficients and thus enhance the selectivity. The iterative process to form two lines of


VIDEO 1. Manipulation of $30-\mu \mathrm{m}$ PS beads. (Top panels) Rearrangement of a random distribution of beads into two vertical lines. (Bottom panel) Writing of the word LIFE with 33 particles initially randomly dispersed. (Video available in the Supplemental Material Ref. [42].) 
particles on this PMMA superstrate is shown at the top of Video 1 still frame, and the final arrangement of 33 beads to form the word LIFE is shown at the bottom of Video 1 still frame. We believe such a rearrangement is figurative of future biological and other microstructures' sequential assembly, where numerous different cells or particles could be arranged in space.

\section{CONCLUSION}

In this paper, we solve an inverse problem to design integrated transducers for arbitrary acoustic fields. We then applied this solution to design an integrated spiraling transducer for acoustical vortices. The insertion of a micromanipulator to force a relative motion between the transducer and the container of the beads allows selective rearrangement of dozens of microspheres. This technology is selective, label-free, biocompatible, flat, easy to miniaturize, and compatible with microfluidic disposable chips. It could finally allow acoustic tweezers to reach the standard laboratory workbench and enable a whole range of alternative biophysical assays and microfabrication processes. This research also opens exciting prospects for cell printing and tissue engineering where dozens of different cell types could be combined and placed accurately to study or print complex biological tissues.

\section{ACKNOWLEDGMENTS}

The authors gratefully acknowledge the PMClab for their support in fabricating the micromanipulation interface. This research was funded by Grant No. ANR-12BS09-0021-01/02 and the Région Nord Pas de Calais.

\section{APPENDIX: CALCULATION OF THE TRANSDUCER GEOMETRY}

In order to obtain Eqs. (2) and (3), we first establish a relation between the wave normal displacement and the prescribed electric field, then assume that the electrodes are good conductors so that they need to be placed along the isophase. In these calculations, we will need to consider the concept of source and receiver in the angular-spectrum viewpoint.

\section{Source and receiver convention for the angular spectrum}

In most cases, the angular spectrum is written in the source convention:

$$
\Xi(\vec{r})=e^{i \omega t} \int_{-\pi}^{\pi} \mathcal{H}_{S}(\psi) e^{-i \omega \overrightarrow{S_{\mathrm{SAW}}} \cdot \vec{r}} d \psi .
$$

However, it also possible to write the same field using the receiver convention:

$$
\Xi(\vec{r})=e^{i \omega t} \int_{-\pi}^{\pi} \mathcal{H}_{R}(\psi) e^{i \omega \overrightarrow{\mathrm{SAW}} \cdot \vec{r}} d \psi .
$$

In both conventions, the acoustic field is a weighted sum of plane waves of amplitude $\mathcal{H}(\psi)$ propagating along a direction $\psi$. However, in the source convention, the waves are diverging from the origin, whereas, in the receiver convention, the waves are converging to the origin. The two conventions are intimately related due the periodicity of $\mathcal{H}$ and $i \omega \overrightarrow{s_{\mathrm{SAW}}} \cdot \vec{r}$ versus $\psi$ :

$$
\mathcal{H}_{S}(\psi)=\mathcal{H}_{R}(\psi+\pi)
$$

which is easily visualized in Fig. 3. An important distinction between source and receiver conventions is the origin of the power. In the first case, the power source is located at the origin, whereas, in the second case, the power source surrounds the origin. In the following, we make all of the calculations in the source convention unless stated otherwise.

\section{Propagation on the substrate}

We consider an arbitrary surface acoustic wave with a slowness $\overrightarrow{s_{\mathrm{SAW}}}(\psi)$ and a piezoelectric coupling given by $a(\psi)=|a(\psi)| e^{i \alpha(\psi)}$. Its normal displacement reads

$$
\Xi^{(0)}(\vec{r})=e^{i \omega(t-\tau)} \int_{-\pi}^{\pi} \mathcal{H}_{S}^{(0)}(\psi) e^{-i \omega \overrightarrow{\mathrm{SAW}}(\psi) \cdot \vec{r}} d \psi
$$

(a)
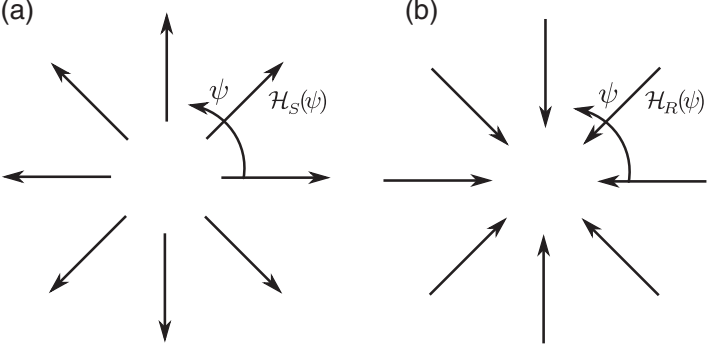

(c)

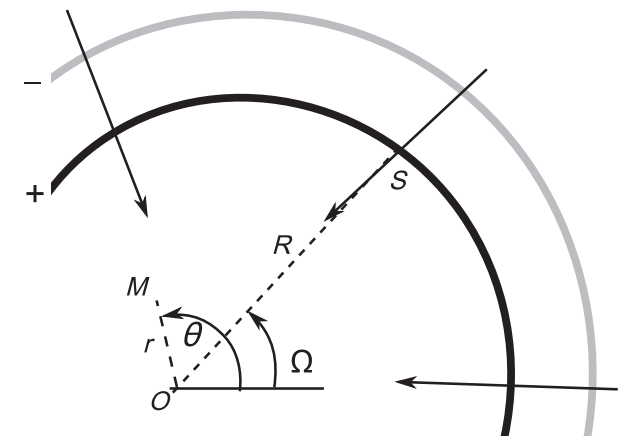

FIG. 3. (a) Source convention (diverging wave front). (b) Receiver convention (converging wave front). (a) and (b) are related by $\mathcal{H}_{R}(\psi)=\mathcal{H}_{S}(\psi+\pi)$. (c) Geometry of the transducer. The electrode is the set of points $S$ of coordinates $(R, \Omega)$ relative to the vortex center $O$. The substrate normal vibrations $\Xi^{(0)}$ and the associated electrical potential fluctuations $\Phi^{(0)}$ are measured at a point $M$ of coordinates $(r, \theta)$. 
In this equation, the delay $\tau$ and the angular spectrum $\mathcal{H}_{S}^{(0)}(\psi)=\mathcal{H}^{(0)}(\psi)=\xi(\psi) e^{i \mu(\psi)}$ are the only degrees of freedom since the slowness is set by the material and its cut.

The aim of the calculation is to find the electric field $\Phi^{(0)}$ associated with the normal displacement $\Xi^{(0)}$. This goal is easily accomplished by considering individual plane waves. For each of them, the normal displacement per unit voltage reads $a(\psi)$. Since $\Xi^{(0)}$ can be resolved as a sum of plane waves over all of the propagation directions $\psi$, we get

$$
\Phi^{(0)}(\vec{r})=e^{i \omega t} \int_{-\pi}^{\pi} \frac{1}{|a(\psi)|} \xi(\psi) e^{i\left[\mu(\psi)-\alpha(\psi)-\omega \overrightarrow{S_{\mathrm{SAW}}}(\psi) \cdot \vec{r}-\psi_{0}^{*}\right]} d \psi
$$

with $\psi_{0}^{*}=\omega \tau$.

According to the aforementioned convention, the origin is taken here as a source. This presumption would be true, for instance, in the case of a small annular transducer that would radiate outwards and if we were interested in this diverging radiation. However, the reverse geometry of a transducer surrounding the origin yields larger power by focusing the surface wave, which makes this design preferable. Consequently, the electroacoustical field should be expressed using the receiver convention:

$$
\Phi^{(0)}(\vec{r})=e^{i \omega t} \int_{-\pi}^{\pi} \frac{1}{|a(\psi+\pi)|} \xi(\psi+\pi) e^{i\left[\mu(\psi+\pi)-\alpha(\psi+\pi)+\omega \overrightarrow{S_{\mathrm{SAW}}}(\psi) \cdot \vec{r}-\psi_{0}^{*}\right]} d \psi .
$$

Equation (A6) can be simplified using the symmetries of the crystal. We introduce the symmetry parameter $b$ such that $a(\psi+\pi)=|a(\psi)| e^{i \alpha(\psi)+i b \pi}$, with the value of $b$ depending upon whether the piezoelectric coupling is symmetric $(b=0)$ or antisymmetric $(b=1)$. This equation yields

$$
\Phi^{(0)}(\vec{r})=e^{i \omega t} \int_{-\pi}^{\pi} \frac{1}{|a(\psi)|} \xi(\psi+\pi) e^{i\left[\mu(\psi+\pi)-\alpha(\psi)+\omega \overrightarrow{\mathrm{SAW}}(\psi) \cdot \vec{r}-\psi_{0}\right]} d \psi,
$$

where we define $\psi_{0}=\psi_{0}^{*}+b \pi$. This integral can be further evaluated in the far-field approximation using the stationary phase. Although the phase reads $\mu(\psi+\pi)-\alpha(\psi)+\omega \overrightarrow{\mathrm{SAW}}(\psi) \cdot \vec{r}-\psi_{0}$, its rapid variations in the far-field are attributable to the $\omega \overrightarrow{s_{\mathrm{SAW}}}(\psi) \cdot \vec{r}=\omega s_{\mathrm{SAW}}(\psi) r \cos (\psi-\theta)=\omega r h(\psi, \theta)$ term. Consequently, the stationary phase is given by $\bar{\psi}(\theta)$ solution of $h^{\prime}(\bar{\psi}, \theta)=0$, with $h^{\prime}(\psi, \theta)=\partial_{\psi}\left[s_{\mathrm{SAW}}(\psi) \cos (\psi-\theta)\right]=0$. This approximation yields

$$
\begin{aligned}
& \bar{\psi}=\theta+\beta, \\
& \beta=\arctan \left(\frac{s_{\text {SAW }}^{\prime}}{s_{\text {SAW }}}\right) .
\end{aligned}
$$

The stationary phase approximation of Eq. (A7) yields

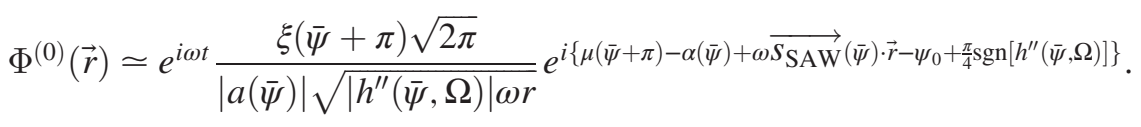

Equation (A10) is similar to the far-field Green's function given in previous works on focused waves by Laude and co-workers $[43,44]$, in which case $\mu=0$ and $\xi=1$ for all $\psi$ 's. We consider a transducer with the positive and negative electrodes running along a polar curve $R(\Omega)$. These electrodes represent isophases of the electrical potential associated with the wave [as given in Eq. (A10)]. In a perfect case, it is possible to apply a voltage $V(\Omega)$ on these electrodes. This configuration yields Eqs. (2) and (3).

\section{Degeneration across a stack of superstrates}

The propagator in Eq. (5) is calculated by angularspectrum propagation. A typical setup composed of $n=3$ superstrates stacked over a substrate is drawn in Fig. 2. Any wave on the substrate reads

$$
\Xi^{(0)}=e^{i \omega t} \int_{-\pi}^{\pi} \mathcal{H}^{(0)}(\psi) e^{-i \omega h(\psi, \theta) r} d \psi,
$$

with $\mathcal{H}^{(0)}$ being its angular spectrum. According to Eq. (8) of an earlier study on vortex degeneration [39], the vibration field in superstrate (i) reads:

$\Xi^{(i)}=e^{i \omega t} \int_{-\pi}^{\pi} \mathcal{H}^{(i-1)}(\psi) e^{-i \omega h(\psi, \theta) r-i \omega s_{z}^{(i)}\left(z-z_{i-1}\right)} d \psi$,

where $z_{i}$ is the height of the upper boundary of the layer $(i)$ and $\mathcal{H}^{(i)}$ is the angular spectrum at this location. The wave must fulfill the dispersion relation of the medium, that is, 
$k_{z}^{(i)}=\omega s_{z}^{(i)}=\sqrt{s^{(i) 2}-s_{r}^{(i) 2}}$. A similar formula applies if the slowness $s^{(i)}$ in the medium (i) depends on $\psi$ and $\sigma$ (if it is anisotropic). In this case, $\vec{s}(\psi, \sigma)$ is a nontrivial slowness surface that depends on the spherical coordinates $\psi$ and $\sigma$. It can be computed in advance by, for instance, numerical simulation. $s_{r}$ and $s_{z}$ are the $r$ and $z$ components of this slowness surface in the cylindrical coordinates. $s_{z}^{(i)}\left[\psi, \sigma_{0}^{(i)}(\psi)\right]$ is then obtained by finding $\sigma_{0}^{(i)}(\psi)$, such that $s_{r}^{(i)}\left(\psi, \sigma_{0}^{(i)}\right)=s_{r}^{(i-1)}\left(\psi, \sigma_{0}^{(i-1)}\right)$.

After traveling through the whole layer (i), this wave hits the next medium. The physical field $\Xi$ is assumed to be continuous across two media (it may be, for instance, the vertical displacement), so that $\left.\Xi^{(i)}\right|_{\text {interface }}=\left.\Xi^{(i+1)}\right|_{\text {interface }}$. Consequently, we get

$$
\mathcal{H}^{(i)}=\mathcal{H}^{(i-1)} e^{-i \omega s_{z}^{(i)}\left(z_{i}-z_{i-1}\right)} .
$$

Since the wave is the same in the close neighborhood of the sides of the interface, $s_{r}$ is also conserved across each interface. Recursively, $s_{r}$ must be the same as in the source medium, which yields $s_{r}=s_{\mathrm{SAW}}$. In anisotropic layers, this equation yields $s_{r}\left[\psi, \phi_{0}^{(i)}(\psi)\right]=s_{\mathrm{SAW}}(\psi)$. A straightforward recurrence on Eq. (A13) then yields Eqs. (5) and (6).

[1] David G. Grier, A revolution in optical manipulation, Nature (London) 424, 810 (2003).

[2] Iwijn De Vlaminck and Cees Dekker, Recent advances in magnetic tweezers, Annu. Rev. Biophys. 41, 453 (2012).

[3] Fernando Martinez-Pedrero and Pietro Tierno, Magnetic Propulsion of Self-Assembled Colloidal Carpets: Efficient Cargo Transport via a Conveyor-Belt Effect, Phys. Rev. Applied 3, 051003 (2015).

[4] Arthur Ashkin, J. M. Dziedzic, J. E. Bjorkholm, and Steven Chu, Observation of a single-beam gradient force optical trap for dielectric particles, Opt. Lett. 11, 288 (1986).

[5] Jeffrey R. Moffitt, Yann R. Chemla, Steven B. Smith, and Carlos Bustamante, Recent advances in optical tweezers, Biochemistry 77, 205 (2008).

[6] Joel Voldman, Electrical forces for microscale cell manipulation, Annu. Rev. Biomed. Eng. 8, 425 (2006).

[7] W. Terence Coakley, David W. Bardsley, Martin A. Grundy, Freidoun Zamani, and David J. Clarke, Cell manipulation in ultrasonic standing wave fields, J. Chem. Technol. Biotechnol. 44, 43 (1989).

[8] R. M. Moroney, R. M. White, and R. T. Howe, Microtransport induced by ultrasonic lamb waves, Appl. Phys. Lett. 59, 774 (1991).

[9] Junru Wu, Acoustical tweezers, J. Acoust. Soc. Am. 89, 2140 (1991).

[10] Jinjie Shi, Daniel Ahmed, Xiaole Mao, Sz-Chin Steven Lin, Aitan Lawit, and Tony Jun Huang, Acoustic tweezers: Patterning cells and microparticles using standing surface acoustic waves (SSAW), Lab Chip 9, 2890 (2009).
[11] Pei Yu Chiou, Aaron T. Ohta, and Ming C. Wu, Massively parallel manipulation of single cells and microparticles using optical images, Nature (London) 436, 370 (2005).

[12] Yanyan Liu and Junhui Hu, Trapping of particles by the leakage of a standing wave ultrasonic field, J. Appl. Phys. 106, 034903 (2009).

[13] Diego Baresch, Jean-Louis Thomas, and Régis Marchiano, Observation of a Single-Beam Gradient Force Acoustical Trap for Elastic Particles: Acoustical Tweezers, Phys. Rev. Lett. 116, 024301 (2016).

[14] Jessica Hultström, Otto Manneberg, Katja Dopf, Hans M. Hertz, Hjalmar Brismar, and Martin Wiklund, Proliferation and viability of adherent cells manipulated by standingwave ultrasound in a microfluidic chip, Ultrasound Med. Biol. 33, 145 (2007).

[15] Otto Manneberg, Bruno Vanherberghen, Jessica Svennebring, Hans M. Hertz, Björn Önfelt, and Martin Wiklund, A three-dimensional ultrasonic cage for characterization of individual cells, Appl. Phys. Lett. 93, 063901 (2008).

[16] Martin Wiklund, Acoustofluidics 12: Biocompatibility and cell viability in microfluidic acoustic resonators, Lab Chip 12, 2018 (2012).

[17] Xiaoyun Ding, Zhangli Peng, Sz-Chin Steven Lin, Michela Geri, Sixing Li, Peng Li, Yuchao Chen, Ming Dao, Subra Suresh, and Tony Jun Huang, Cell separation using tiltedangle standing surface acoustic waves, Proc. Natl. Acad. Sci. U.S.A. 111, 12992 (2014).

[18] Feng Guo, Yuliang Xie, Sixing Li, James Lata, Liqiang Ren, Zhangming Mao, Baiyang Ren, Mengxi Wu, Adem Ozcelik, and Tony Jun Huang, Reusable acoustic tweezers for disposable devices, Lab Chip 15, 4517 (2015).

[19] Xiaoyun Ding, Sz-Chin Steven Lin, Brian Kiraly, Hongjun Yue, Sixing Li, I.-Kao Chiang, Jinjie Shi, Stephen J. Benkovic, and Tony Jun Huang, On-chip manipulation of single microparticles, cells, and organisms using surface acoustic waves, Proc. Natl. Acad. Sci. U.S.A. 109, 11105 (2012).

[20] S. B. Q. Tran, Philippe Marmottant, and Pierre Thibault, Fast acoustic tweezers for the two-dimensional manipulation of individual particles in microfluidic channels, Appl. Phys. Lett. 101, 114103 (2012).

[21] David J. Collins, Belinda Morahan, Jose Garcia-Bustos, Christian Doerig, Magdalena Plebanski, and Adrian Neild, Two-dimensional single-cell patterning with one cell per well driven by surface acoustic waves, Nat. Commun. 6, 8686 (2015).

[22] Jungwoo Lee, Shia-Yen Teh, Abraham Lee, Hyung Ham Kim, Changyang Lee, and K. Kirk Shung, Single beam acoustic trapping, Appl. Phys. Lett. 95, 073701 (2009).

[23] Ying Li, Changyang Lee, Ruimin Chen, Qifa Zhou, and K. Kirk Shung, A feasibility study of in vivo applications of single beam acoustic tweezers, Appl. Phys. Lett. 105, 173701 (2014).

[24] Jae Youn Hwang, Chi Woo Yoon, Hae Gyun Lim, Jin Man Park, Sangpil Yoon, Jungwoo Lee, and K. Kirk Shung, Acoustic tweezers for studying intracellular calcium signaling in SKBR-3 human breast cancer cells, Ultrasonics 63, 94 (2015).

[25] Jae Youn Hwang, Jihun Kim, Jin Man Park, Changyang Lee, Hayong Jung, Jungwoo Lee, and K. Kirk Shung, Cell deformation by single-beam acoustic trapping: A promising 
tool for measurements of cell mechanics, Sci. Rep. 6, 27238 (2016).

[26] Deny Hartono, Yang Liu, Pei Lin Tan, Xin Yi Sherlene Then, Lin-Yue Lanry Yung, and Kian-Meng Lim, On-chip measurements of cell compressibility via acoustic radiation, Lab Chip 11, 4072 (2011).

[27] Mikkel Settnes and Henrik Bruus, Forces acting on a small particle in an acoustical field in a viscous fluid, Phys. Rev. E 85, 016327 (2012).

[28] Jonas T. Karlsen and Henrik Bruus, Forces acting on a small particle in an acoustical field in a thermoviscous fluid, Phys. Rev. E 92, 043010 (2015).

[29] Philip L. Marston, Axial radiation force of a bessel beam on a sphere and direction reversal of the force, J. Acoust. Soc. Am. 120, 3518 (2006).

[30] Asier Marzo, Sue Ann Seah, Bruce W. Drinkwater, Deepak Ranjan Sahoo, Benjamin Long, and Sriram Subramanian, Holographic acoustic elements for manipulation of levitated objects, Nat. Commun. 6, 8661 (2015).

[31] Charles R. P. Courtney, Christine E. M. Demore, Hongxiao Wu, Alon Grinenko, Paul D. Wilcox, Sandy Cochran, and Bruce W. Drinkwater, Independent trapping and manipulation of microparticles using dexterous acoustic tweezers, Appl. Phys. Lett. 104, 154103 (2014).

[32] Brian T. Hefner and Philip L. Marston, An acoustical helicoidal wave transducer with applications for the alignment of ultrasonic and underwater systems, J. Acoust. Soc. Am. 106, 3313 (1999).

[33] Jean-Louis Thomas and Régis Marchiano, Pseudo Angular Momentum and Topological Charge Conservation for Nonlinear Acoustical Vortices, Phys. Rev. Lett. 91, 244302 (2003).

[34] Noé Jiménez, Rubén Picó, Víctor Sánchez-Morcillo, Vicent Romero-García, Lluís M. García-Raffi, and Kestutis Staliunas, Formation of high-order acoustic bessel beams by spiral diffraction gratings, Phys. Rev. E 94, 053004 (2016).

[35] Christina J. Naify, Charles A. Rohde, Theodore P. Martin, Michael Nicholas, Matthew D. Guild, and Gregory J. Orris, Generation of topologically diverse acoustic vortex beams using a compact metamaterial aperture, Appl. Phys. Lett. 108, 223503 (2016).
[36] Kai Melde, Andrew G. Mark, Tian Qiu, and Peer Fischer, Holograms for acoustics, Nature (London) 537, 518 (2016).

[37] A. Riaud, M. Baudoin, J. L. Thomas, and O. Bou Matar, SAW synthesis with IDTs array and the inverse filter: Toward a versatile saw toolbox for microfluidic and biological applications, IEEE Trans. Ultrason. Ferroelectr. Freq. Control 63, 1601 (2016).

[38] Antoine Riaud, Jean-Louis Thomas, Eric Charron, Adrien Bussonnière, Olivier Bou Matar, and Michael Baudoin, Anisotropic Swirling Surface Acoustic Waves from Inverse Filtering for On-Chip Generation of Acoustic Vortices, Phys. Rev. Applied 4, 034004 (2015).

[39] Antoine Riaud, Jean-Louis Thomas, Michael Baudoin, and Olivier Bou Matar, Taming the degeneration of bessel beams at an anisotropic-isotropic interface: Toward threedimensional control of confined vortical waves, Phys. Rev. E 92, 063201 (2015).

[40] Olivier Bou Matar, Noura Gasmi, Huan Zhou, Marc Goueygou, and Abdelkrim Talbi, Legendre and Laguerre polynomial approach for modeling of wave propagation in layered magneto-electro-elastic media, J. Acoust. Soc. Am. 133, 1415 (2013).

[41] D. Royer and E. Dieulesaint, Elastic Waves in Solids I: Free and Guided Propagation (Springer Science+Business Media, New York, 1999).

[42] See Supplemental Material at http://link.aps.org/ supplemental/10.1103/PhysRevApplied.7.024007 for a detailed analysis.

[43] V. Laude, D. Gerard, N. Khelfaoui, C. F. Jerez-Hanckes, S. Benchabane, and A. Khelif, Subwavelength focusing of surface acoustic waves generated by an annular interdigital transducer, Appl. Phys. Lett. 92, 094104 (2008).

[44] V. Laude, C. F. Jerez-Hanckes, and S. Ballandras, Surface Green's function of a piezoelectric half-space, IEEE Trans. Ultrason. Ferroelectr. Freq. Control 53, 420 (2006).

[45] L. P. Gor'Kov, On the forces acting on a small particle in an acoustical field in an ideal fluid, Dokl. Phys. 6, 773 (1962).

[46] K. T. Gahagan and G. A. Swartzlander, Optical vortex trapping of particles, Opt. Lett. 21, 827 (1996). 